\title{
Dynamical behaviour of a compound elastic pendulum
}

\author{
Svetoslav Nikolov ${ }^{1,2, *}$ and Daniela Zaharieva ${ }^{2}$ \\ ${ }^{1}$ Institute of Mechanics-BAS, Biomechanics Department, Acad. G. Bonchev Str., Bl. 4, 1113 Sofia, \\ Bulgaria \\ ${ }^{2}$ University of Transport, Mechanics Department, G. Milev Str. 158, 1574 Sofia, Bulgaria
}

\begin{abstract}
The aim of the paper is a comprehensive study of the compound elastic pendulum (CEP) with two degrees of freedom to point out the main complex (chaotic) dynamics that it can exhibit. The simplest way to find complex behavior in a nonintegrable Hamiltonian system is firstly to look for homoclinic (heteroclinic) orbit(s). Here, under suitable assumptions, we detect the existence of a homoclinic orbit of CEP and present the equation for it. Moreover, we show that for any value of the small parameter the system has a hyperbolic periodic orbit, whose invariant manifolds intersect themselves transversally.
\end{abstract}

\section{Introduction}

Nonlinear dynamics studies some conditions that are not observed in linear systems. Nonlinear behavior (movement and evolution) is typical for different dynamical systems. The investigation of nonlinear mechanical systems is an important and very active area [1]. For example, the pendulum is interesting as a paradigm of contemporary dynamics and, more importantly, since the differential equation of the pendulum is frequently encountered in various branches of modern mechanics $[2,3]$.

The study of complex phenomena in deterministic nonlinear dynamical systems has attracted the attention of applied scientists. This study poses two fundamental questions: 1) by what mechanisms does chaos occur and 2) how can one predict when chaos will occur in a specific dynamical system?

Dynamical systems can be separated into two main kinds: i) systems with conservation of phase volume and ii) systems with decrease of phase volume - called dissipative systems. Systems from kind (i) can be Hamiltonian and non-Hamiltonian systems. Systems are called Hamiltonian if their equations can be written in canonical form by means of a Hamiltonian $H(q, p, t)$, where $q$ and $p$ are generalized coordinates and momenta, and $t$ is the time.

Generally, bifurcations can be local or global. Global bifurcations are qualitative change in the orbit structure of an extended region of phase space. Typical examples are homoclinic and heteroclinic bifurcations.

\footnotetext{
* Corresponding author: S.Nikolov@imbm.bas.bg
} 
In recent years, it has become apparent that homoclinic and heteroclinic orbits are often the mechanism for the chaos (or transient to chaos) in different kinds of dynamical systems - mechanical, biological and et al. [4-8].

Definition 1. (Wiggins [9]) Let $\Phi$ be an invariant set of a dynamical - map or flow. Let $p$ be a point in the phase (space) of the dynamical system and the orbit of $p$ approaches $\Phi$ asymptotically as $t \rightarrow-\infty$ and $\Phi$ asymptotically as $t \rightarrow+\infty$. Then the orbit of $p$ is said to be homoclinic to $\Phi$.

Definition 2.(Wiggins [9]) Let $\Phi_{1}$ and $\Phi_{2}$ be disjoint invariant sets of a dynamical system, and the orbit of $p$ approaches $\Phi_{1}$ asymptotically as $t \rightarrow-\infty$ and $\Phi_{2}$ asymptotically as $t \rightarrow+\infty$. Then the orbit of $p$ is said to be heteroclinic to $\Phi_{1}$ and $\Phi_{2}$.

Definitions 1 and 2 (D.1 and D2) are too general, as in case that $\Phi, \Phi_{1}$ and $\Phi_{2}$ passes stable and unstable manifolds (e.g. they are hyperbolic or normally hyperbolic), they can be alternately stated as follows.

Definition 3. Let $\Phi, \Phi_{1}, \Phi_{2}$ and $p$ be denoted as D.1 and D.2. If $p$ lies in both the stable and unstable manifolds of $\Phi$, then the orbit $p$ is said to be homoclinic to $\Phi$.

If $p$ lies in the unstable manifold of $\Phi_{1}$ and the stable manifold of $\Phi_{2}$, then the orbit of $p$ is heteroclinic to $\Phi_{1}$ and $\Phi_{2}$.

Note here that the point $p$ is a homoclinic (resp. heteroclinic) point.

For mechanical systems which are modeled by Hamiltonian equations of motion, integrability is a central problem. Most Hamiltonian systems are not integrable but if a Hamiltonian dynamical system is integrable, then its first integrals provide a description of all features of the system [10-12].

It is well-known that all Hamiltonian systems with one degree of freedom and analytical Hamiltonian functions are integrable, and there is no splitting of separatrices. If separatrices of two saddles coincide to a form of heteroclinic orbit then we have a connection bifurcation. This is a global bifurcation as a necessary condition is that the two saddles have the same energy [13]. On the other hand, integrability is not a typical property of Hamiltonian systems with more than one degree of freedom. The phase spaces of such systems are difficult to visualize and a lower dimensional representation of the qualitative behavior of the system must be sought.

The importance of the study of homoclinic (heteroclinic) orbits in nonintegrable Hamiltonian systems has been appreciated since the time of Poincare [14, 15]. Now, it is fairly well-known that Hamiltonian systems with two (or more) degrees of freedom are generally nonintegrable, which usually involves chaotic dynamics. All bounded trajectories of an integrable Hamiltonian system with $n=2$ degrees of freedom evolve in the $2 n$ phase space on manifolds which are diffeomorphic (equivalent) to 2-tori [16]. If integrability is broken, KAM theory guarantees that tori (from resonances) will survive and the motion bears a nonregular (chaotic) character [9]. Furthermore, in Hamiltonian systems with two degrees of freedom there are five distinct cases to consider according to the different possibilities for the eigenvalues of the linearized vector field at a hyperbolic fixed point [9]. They are:
Real eigenvalues
$\left\{\begin{array}{l}\text { 1) } \chi_{1}, \chi_{2}>0 ; \chi_{3}, \chi_{4}<0 . \\ \text { 2) } \chi_{1}>0 ; \chi_{2}, \chi_{3}, \chi_{4}<0 .\end{array}\right.$
Complex
eigenvalues
3) $n_{1} \pm i m_{1}, n_{2} \pm i m_{2} ; n_{1}>0$,
$n_{2}<0, m_{1}, m_{2} \neq 0$. 


$$
\text { Real and complex eigenvalues }\left\{\begin{array}{l}
\text { 4) } n_{1} \pm i m_{1}, \chi_{1}, \chi_{2}>0 ; n_{1}<0, m_{1} \neq 0 . \\
5) n_{1} \pm i m_{1}, \chi_{1}<0, \chi_{2}>0 ; n_{1}<0, m_{1} \neq 0 .
\end{array}\right.
$$

Other cases may be obtained from these via time reversal. If nonhyperbolic fixed points are considered, then even more cases must be considered.

In generic systems with two degrees of freedom the following types of bifurcations of periodic and homoclinic orbits are possible: i) the periodic saddle-centre bifurcation $[5,6$, 13] and ii) the Hamiltonian period-doubling bifurcation. In case (i), under variation of the energy a hyperbolic and elliptic periodic orbit meet at a parabolic periodic orbit with all Floquet multipliers equal to one. In case (ii), under variation of the energy an elliptic periodic orbit turns hyperbolic when passing through a parabolic periodic orbit with Floquet multipliers minus one. Simultaneously, from the parabolic periodic orbit, a family of periodic orbits with the double period emerges, inheriting the normal linear behavior from the initial periodic orbit. The behavior near the phase-space curves separating regions with different type of motion (so called separatrices) is generic for nonlinear Hamiltonian systems.

In this paper we consider a compound elastic pendulum (CEP) with two degrees of freedom- see Figure 1. For this pendulum the kinetic energy $T$ and the potential energy $U$ have the form

$$
\begin{aligned}
& T=\frac{m_{1}}{2} L^{2} \dot{\theta}^{2}+\frac{m_{2}}{2}\left[(l-\xi)^{2} \dot{\theta}^{2}+\dot{\xi}^{2}\right], \\
& U=-m_{1} g L(1-\cos \theta)-m_{2} g(l-\xi)(1-\cos \theta)+\frac{c}{2} \xi^{2},
\end{aligned}
$$

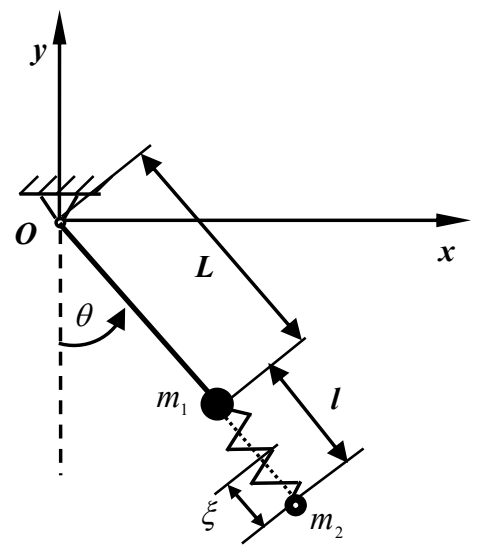

Fig. 1. The mass-spring pendulum system.

where $m_{1}$ and $m_{2}$ are the masses, $\theta$ is the corresponding angle of the pendulum with the gravity vertical (defined $\bmod 2 \pi$ ), $\xi$ is the deviation of mass $m_{2}$ from equilibrium state, $l$ is the distance from mass $m_{1}$ to equilibrium of mass $m_{2}$ (while both rod and spring are massless), $L$ is the length of the pendulum $\left(O m_{1}\right), c$ is the spring constant and $g$ is the acceleration of the gravity.

Here, we are interested in the dynamical behavior of the pendulum shown in Figure 1, and specially in the homoclinic orbit equation for it.

The paper is organized as follows: In Section two, we obtain the analytical results for the Hamiltonian of the compound elastic pendulum. In Section 3, we present the analytical results for homoclinic loop of CEP. Finally, Section 4 summarizes our results. 


\section{Hamiltonian form of the compound elastic pendulum}

In the Hamiltonian formulation, the state of a system is characterized not only by its positions $q_{i}(i=1, \ldots, n)$, but also by its momenta $p_{i}$. Thus, to obtain the Hamiltonian $H\left(\theta, p_{1}, \xi, p_{2}\right)$ of CEP, we firstly pass to the canonical momentum representation

$$
p_{1}=\frac{\partial T}{\partial \dot{\theta}} \quad, \quad p_{2}=\frac{\partial T}{\partial \dot{\xi}} .
$$

Hence, the autonomous Hamiltonian has the form

$$
\begin{aligned}
& H\left(\theta, p_{1}, \xi, p_{2}\right)=T-U=\frac{1}{2 m_{2} \psi}\left[m_{2} p_{1}^{2}+\psi p_{2}^{2}\right]+ \\
& \quad+m_{1} g L(1-\cos \theta)+m_{2} g(l-\xi)(1-\cos \theta)-\frac{c}{2} \xi^{2},
\end{aligned}
$$

where $\psi=m_{1} L^{2}+m_{2}(l-\xi)^{2}$. The time-evolution of the system is then governed by equations of motion

$$
\begin{aligned}
& \dot{\theta}=\frac{\partial H}{\partial p_{1}}, \dot{\xi}=\frac{\partial H}{\partial p_{2}} \\
& \dot{p}_{1}=-\frac{\partial H}{\partial \theta}, \dot{p}_{2}=-\frac{\partial H}{\partial \xi} .
\end{aligned}
$$

An immediate consequence is

$$
\dot{H}=\frac{\partial H}{\partial \theta} \dot{\theta}+\frac{\partial H}{\partial p_{1}} \dot{p}_{1}+\frac{\partial H}{\partial \xi} \dot{\xi}+\frac{\partial H}{\partial p_{2}} \dot{p}_{2}=0,
$$

the conservation of energy. Here we note that the system (4) is with two degrees of freedom and belongs to the class of nonintegrable Hamiltonian systems. The four-dimensional phase space $\mathrm{Z}=\left\{\left(\theta, \xi, p_{1}, p_{2}\right)\right\}$ is characterized by an extremely complex behavior of the phase trajectories and by existence of instability zones in which the motion has chaotic (nonregular) character $[9,16]$.

The fixed points of the system (4) in the $\left(\theta, \xi, p_{1}, p_{2}\right)$ plane defined as

$$
\frac{\partial H}{\partial p_{1}}=\frac{\partial H}{\partial p_{2}}=\frac{\partial H}{\partial \theta}=\frac{\partial H}{\partial \xi}=0
$$

are

$$
\bar{p}_{1}=\bar{p}_{2}=0, \bar{\theta}=k \pi(k=0, \pm 1, \pm 2, \ldots), \bar{\xi}^{(1)}=0, \bar{\xi}^{(2)}=-\frac{2 m_{2} g}{c} .
$$

According to [9], the law of energy conservation $E=T-U$ defines an invariant three-dimensional hypersurface $H=E$ about the system phase flow. Here we are interested in a special phase curve- homoclinic orbit, lying in the three-dimensional manifold with level of energy $E_{h}$. This level corresponds to the 'upper' position (the masses standing upright vertically) of the compound elastic pendulum, i.e. $\bar{\theta}= \pm \pi$, $\bar{\xi}=-\frac{2 m_{2} g}{c}, \bar{p}_{1}=\bar{p}_{2}=0$. Hence, the manifold $E_{h}$ has the form

$$
H\left(\bar{\theta}, \bar{p}_{1}, \bar{\xi}, \bar{p}_{2}\right)=E_{h}=2 g\left[m_{1} L+m_{2}\left(l+\frac{m_{2} g}{c}\right)\right] .
$$




\section{Homoclinic solution}

In the previous section, we obtained some analytical results that we shall use in our calculations so as to find a homoclinic solution (orbit) $\left(\theta_{h}(t), p_{1 h}(t), \xi_{h}(t), p_{2 h}(t)\right)$ in the three-dimensional manifold $E_{h}$. Note that this homoclinic solution is transversal intersection of the two-dimensional invariant surfaces $W^{s}$ (stable manifold) and $W^{u}$ (unstable manifold) of the hyperbolic fixed point $\bar{\theta}= \pm \pi, \bar{\xi}=-\frac{2 m_{2} g}{c}, \bar{p}_{1}=\bar{p}_{2}=0$. In our considerations, we introduce the small parameter $\varepsilon=m_{2} / m_{1}<<1$, i.e. $0<m_{2}<m_{1}$.

To find the equation of the homoclinic orbit we use the Lindstedt-Poincare perturbation method (procedure). For a small perturbation parameter, $\varepsilon$, the system (4) can be written in the form

$$
\begin{aligned}
& \dot{\theta}=\frac{1}{m_{1}\left[L^{2}+\varepsilon(l-\xi)^{2}\right]_{1}} \quad, \quad \dot{\xi}=\frac{1}{m_{1} \varepsilon} p_{2}, \\
& \dot{p}_{1}=-m_{1} g \sin \theta[L+\varepsilon(l-\xi)], \\
& \dot{p}_{2}=-\frac{\varepsilon(l-\xi)}{m_{1}\left[L^{2}+\varepsilon(l-\xi)^{2}\right]^{2}} p_{1}^{2}+m_{1} g \varepsilon(1-\cos \theta)+c \xi .
\end{aligned}
$$

The homoclinic solution (trajectory) of system (9) satisfies the boundary conditions

$$
\begin{aligned}
& \theta \rightarrow \pi, \xi \rightarrow-\frac{2 m_{2} g}{c}, p_{1} \rightarrow 0, p_{2} \rightarrow 0, \text { for } t \rightarrow+\infty \\
& \theta \rightarrow-\pi, \xi \rightarrow-\frac{2 m_{2} g}{c}, p_{1} \rightarrow 0, p_{2} \rightarrow 0, \text { for } t \rightarrow-\infty .
\end{aligned}
$$

The functions $\theta, \xi, p_{1}$ and $p_{2}$ as series in powers of $\varepsilon$ can be presented in the form

$$
\begin{aligned}
& \theta=\theta_{0}+\varepsilon \theta_{1}+\varepsilon^{2} \theta_{2}+\ldots \quad, \quad \xi=\xi_{0}+\varepsilon \xi_{1}+\varepsilon^{2} \xi_{2}+\ldots \\
& p_{1}=p_{10}+\varepsilon p_{11}+\varepsilon^{2} p_{12}+\ldots \quad, \quad p_{2}=p_{20}+\varepsilon p_{21}+\varepsilon^{2} p_{22}+\ldots
\end{aligned}
$$

After substituting of (11) into (9) and accomplishing some transformations and analytical calculations we obtain the following equations for the functions $\theta_{0}, \xi_{1}, p_{10}, p_{22}$

$$
\begin{aligned}
& \dot{\theta}_{0}=\frac{1}{m_{1} L^{2}} p_{10} \quad, \quad \dot{\xi}_{1}=\frac{1}{m_{1}} p_{22}, \quad \dot{p}_{10}=-m_{1} g L \sin \theta_{0}, \\
& \dot{p}_{22}=\frac{1}{m_{1} L^{4}} \xi_{1} p_{10}^{2}+2 \frac{m_{1} g l^{2}}{L^{2}}\left(1-\cos \theta_{0}\right)+2 \frac{c l^{2}}{L^{2}} \xi_{1}+c \xi_{2},
\end{aligned}
$$

where $\xi_{2}=$ const . Here we note that $\xi_{0}, p_{20}$ and $p_{21}$ are equal to zero.

The function $\theta_{0}$ is defined by the equation

$$
\ddot{\theta}_{0}+\omega^{2} \sin \theta_{0}=0
$$

yielding the homoclinic solution

$$
\theta_{0}(t)= \pm 2 \operatorname{arctg}(\operatorname{sh}(\omega t))
$$

where $\omega^{2}=g / L$ (for details see Appendix 1). Hence, for function $p_{10}$ we have

$$
p_{10}(t)= \pm m_{1} L^{2} \frac{2 \omega}{c h(\omega t)} .
$$


The solution (15) is called also a soliton (self-dependent wave) - see Fig. 2. It is wellknown that solitons are nonlinear waves which after a fully nonlinear interaction do not change their speed and shape. For the function $\xi_{1}$ respectively we have

$$
\ddot{\xi}_{1}=a(t) \xi_{1}+b\left(1-\cos \theta_{0}\right)+\alpha,
$$

where $a(t)=\left(\frac{2 \omega}{c h(\omega t)}\right)^{2}+\frac{2 c l^{2}}{m_{1} L^{2}}, b=\frac{2 g l^{2}}{L^{2}}$ and $\alpha=\frac{c}{m_{1}} \xi_{2}$.

The equation (16) is investigated in the Appendix 2 of this work. Thus, after acceptance that $a(t)=A(t)=$ const $.>0, \theta_{0} \in(0, \pi]$ and $\xi_{2}=0$, we obtain (without loss of generality) that (16) has a homoclinic solution in the form

$$
\xi_{1}(t)=C_{1} \operatorname{sh}(t \sqrt{A})+C_{2} \operatorname{ch}(t \sqrt{A})-C_{3},
$$

where $C_{1}=\frac{1}{\sqrt{A}} \dot{\xi}_{1}\left(t_{0}\right), C_{2}=\xi_{1}\left(t_{0}\right)$ and $C_{3}=\frac{B}{A}=\frac{m_{1} g l^{2} c h^{2}\left(\omega t_{0}\right)\left(1-\cos \theta_{0}\right)}{2 m_{1} \omega^{2} L^{2}+c l^{2} c h^{2}\left(\omega t_{0}\right)}$ are constants.

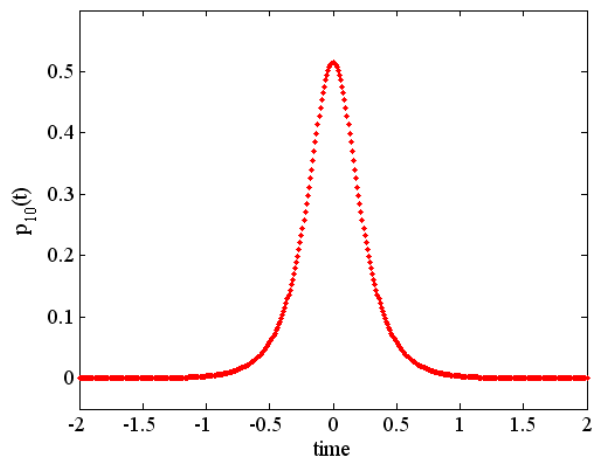

Fig. 2. A soliton solution for the velocity of the separatrice Eq. (15) (in $\left(\theta_{0}, p_{10}\right)$ plane) when: $L=0.3[\mathrm{~m}], m_{1}=0.5[\mathrm{~kg}]$ and time $t \in[-2,2]$ is in seconds. Note that when we have sign "+" into (15), then the soliton motion is to the right (upper part of the separatrice).

For the function $p_{22}$, using (15) and (17) (when $B=0$ and $p_{10}(t)=p_{10}^{+}(t)$ ), we obtain the following first order differential equation

$$
\dot{p}_{22}=m_{1} \widetilde{A}\left(C_{1} \operatorname{sh}(t \sqrt{\tilde{A}})+C_{2} \operatorname{ch}(t \sqrt{\widetilde{A}})\right) .
$$

The homoclinic solution of the equation (18) has the form

$$
p_{22}=A_{1} \operatorname{ch}(t \sqrt{\tilde{A}})-A_{2} \operatorname{sh}(t \sqrt{\tilde{A}}),
$$

where

$$
A_{1}=m_{1} C_{1} \sqrt{\tilde{A}}, A_{2}=m_{1} C_{2} \sqrt{\widetilde{A}}, \tilde{A}=\left(\frac{2 \omega}{\operatorname{ch}(\omega t)}\right)
$$

\section{Numerical calculations}

In this section we investigate numerically the system (9) in terms of the Poincare section. The first step is finding the hyperbolic periodic points(s) for the Poincare map $[17,18]$. The approximate position of some hyperbolic point, for $\varepsilon=0.2$, was obtained visually from the picture of the phase portrait. The second step is the construction of separatrices. In this 
connection, the pictures of corresponding stable $W^{s}$ and unstable manifolds $W^{u}$ are shown in Figure 3. The last step is to prove the existence of homoclinic transversal intersection(s). If the curves $W^{s}$ and $W^{u}$ are not tangent at a homoclinic point $q\left(q \in W^{s} \cap W^{u}\right)$, then the intersection is transversal, and q is said to be a transversal homoclinic orbit. Here we note that this step is the central and most complicated part of the procedure for the proof of the existence of chaotic motion, and will be considered in our forthcoming work.
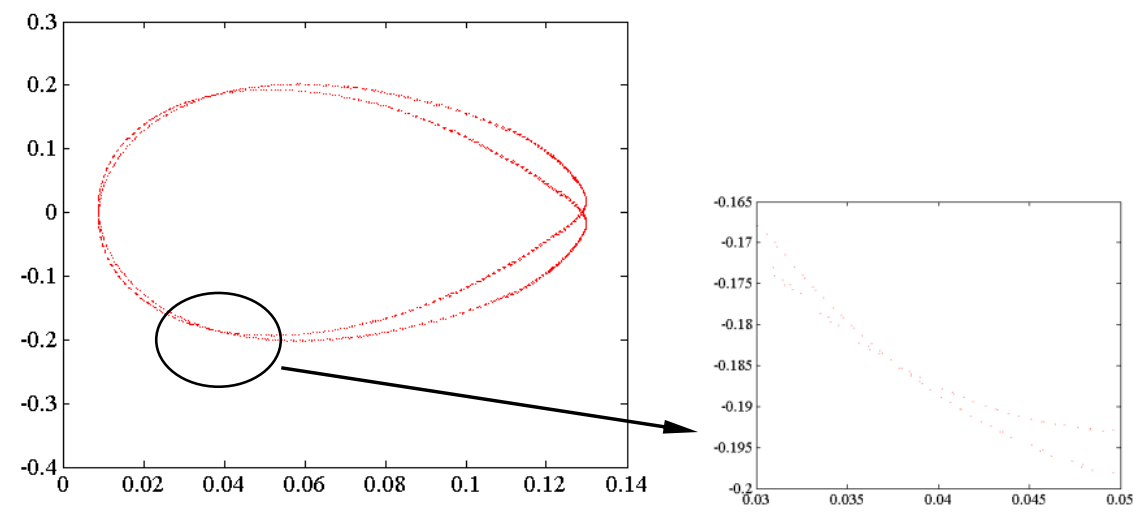

Fig.3. Poincare section and transverse intersection of stable and unstable manifolds of the system (9) for the perturbation $\varepsilon=0.2$ and $c=32.15[\mathrm{~N} / \mathrm{m}]$.

\section{Conclusions}

In this paper were presented some analytical and numerical results for existence of a homoclinic loop in Hamiltonian systems with two degrees of freedom - compound elastic pendulum (CEP). It is to be noted that the main conjecture from the results obtained in analytical calculations is that CEP with two degrees of freedom is nonintegrable for all nontrivial (non equal to zero or infinity) values of the parameters.

For Hamiltonian systems, there are two types of hyperbolic fixed points: 1) saddlefocus and 2) saddle. If these systems are from fourth order, the homoclinic orbit lies in the transversal interaction of the stable $W^{s}$ and unstable $W^{u}$ manifolds of the origin. For both types of fixed points, horseshoes exist on the level sets $H^{-1}(\varepsilon)$ for $\varepsilon$ sufficiently small due to the structural stability of the horseshoes. Detecting of homoclinic (heteroclinic) orbits in a nonintegrable Hamiltonian system is not an easy task, but the existence of horseshoes forms conditions for the developing of chaotic behavior [19]. In this connection, in section 4, we show that for any value of the small parameter $\varepsilon$ the system CEP has a hyperbolic periodic trajectory, whose invariant manifolds intersect transversally.

This work was supported by grant No 110/18.04.2017 of the University of Transport "T. Kableshkov" Sofia, Bulgaria.

\section{References}

1. M. Brin, G. Stuck, Introduction to dynamical systems (CUP, Cambridge, 2003)

2. S. Nikolov, Ju. Genov, N. Nachev, Mechanics, Transport, Commun. 8, 0472 (2010)

3. L. Shilnikov, A. Shilnikov, D. Turaev, L. Chua, Methods of qualitative theory in nonlinear dynamics (Part II, World Scientific, Singapore, 2001) 
4. S. Nikolov, Mechanics, Transport, Commun. 10, 0507 (2012)

5. V. Afraimovich, S. Gonchenko, L. Lerman, A. Shilnikov, D. Turaev., Regular and Chaotic Dynamics 19, 435-460 (2014)

6. R. Driesse, A. Homburg, J. of Differential Equations 246, 2681-2705 (2009)

7. S. Nikolov, D. Zaharieva, Mechanics, Transport, Commun. 14, 1352 (2016)

8. S. Nikolov, O. Wolkenhauer, J. Vera, Molecular BioSystems 10, 172-179 (2014)

9. S. Wiggins, Global bifurcations and chaos. Analytical methods (Springer-Verlag, NY, 1988)

10. M. Brack, K. Tanaka, Physical Review E 77, 046205 (2008)

11. G. Villasi, Hamiltonian dynamics (World Scientific, Singapore, 2001)

12. J. Lowenstein, Essentials of Hamiltonian dynamics (CUP, NY, 2012)

13. H. Hansmann, Local and semi-local bifurcations in Hamiltonian dynamical systems (Springer, Berlin, 2007)

14. H. Poincare, Les methodes nouvelles de la mecanique celeste, 1-3 (Gauthier-Villars, $1892 ; 1893 ; 1899)$

15. R. MacKay, J. Meiss, Hamiltonian dynamical systems (Adam Hulger, Boston, 1987).

16. V. Arnold, Mathematical methods of classical mechanics (Springer-Verlag, Heidelberg, 1978)

17. V. Gelfreich, D. Sharomov, Physics Letters A, 197, 139-146 (1995)

18. A. Ivanov, J. of Physics A 34, 11011-11031 (2001)

19. G. Van Der Heijden, K. Yagasaki, Z. Angew. Math. Phys. 65, 221-240 (2014)

20. A. King, A., J. Billingham, S. Otto, Differential equations. Linear, nonlinear, ordinary, partial (Cambridge University Press, NY, 2003)

21. H. Josephs, R. Huston, Dynamic of mechanical systems (CRC Press, NY, 2002)

22. G. Murphy, Ordinary differential equations and their solutions (Van Nostrand, NY, 1960)

23. D. Zwillinger, Handbook of differential equations (Academic Press, NY, 1997)

24. G. Baker, J. Blackburn, The pendulum. A case study in physics (Oxford University Press, NY, 2005)

\section{Appendix}

\section{A.1 Homoclinic solution of (13)}

Let us consider

$$
\frac{d^{2} \theta}{d t^{2}}+\omega^{2} \sin \theta=0
$$

where $\omega^{2}=g / L$ and $t$ is time. For oscillations of small amplitude (i.e. $\theta<6^{0} \div 10^{\circ}$, so that $\sin \theta \approx \theta$ ) we obtain simple harmonic motion in the form

$$
\frac{d^{2} \theta}{d t^{2}}+\omega^{2} \theta=0
$$

Here, the general solution of (A.2) is

$$
\theta=A_{1} \sin \omega t+B_{1} \cos \omega t
$$

where $A_{1}$ and $B_{1}$ are constants that can be fixed from the initial values of $\theta$ and $\dot{\theta}=d \theta / d t$. However, if $\theta$ is not small $\left(\theta>10^{\circ}\right)$ the linearization approximation is no longer valid. Thus, we must study the full equation of motion (A.1), which is nonlinear. It is well-known that, in general, nonlinear differential equations cannot be solved analytically, but for equation (A.1) (where the first derivative $d \theta / d t$ does not appear 
explicitly), an analytical solution is available [20]. Treating $\dot{\theta}$ as a function of $\theta$ instead of $t$, we have

$$
\frac{d^{2} \theta}{d t^{2}}=\frac{d}{d t}\left(\frac{d \theta}{d t}\right)=\frac{d \dot{\theta}}{d t}=\frac{d \theta}{d t} \frac{d \dot{\theta}}{d \theta}=\dot{\theta} \frac{d \dot{\theta}}{d \theta}=\frac{d}{d \theta}\left(\frac{1}{2} \dot{\theta}^{2}\right) .
$$

Hence, for (A.1) we can write

$$
\frac{d}{d \theta}\left(\frac{1}{2} \dot{\theta}^{2}\right)=-\omega^{2} \sin \theta
$$

which we can integrate once to give

$$
\frac{1}{2} \dot{\theta}^{2}=\omega^{2} \cos \theta+c_{1},
$$

where $c_{1}$ is a constant that can be evaluated by initial conditions, i.e. when $t=0, \theta=\theta_{0}=$ const . and $\dot{\theta}=0$. Thus, using also that $\omega^{2}=g / L$, the first integral of (A.6) becomes

$$
\frac{1}{2} m_{1} L^{2} \dot{\theta}^{2}-m_{1} g L \cos \theta=E=-\frac{g}{L} \cos \theta_{0},
$$

where $E$ is a statement of conservation of energy. From (A.7) we can see that

$$
\frac{d \theta}{d t}= \pm \sqrt{\frac{2 E}{m_{1} L^{2}}+\frac{2 g}{L} \cos \theta}= \pm \sqrt{\frac{2 g}{L}\left(\cos \theta-\cos \theta_{0}\right)} .
$$

Thus, we have a nonlinear first-order differential equation to solve. By separating the variables, we have

$$
d t= \pm \sqrt{\frac{L / 2 g}{\cos \theta-\cos \theta_{0}}} d \theta .
$$

By integration of (A.9) we obtain

$$
t=\sqrt{\frac{L}{2 g}} \int_{\theta}^{\theta_{0}} \sqrt{\frac{1}{\cos \theta-\cos \theta_{0}}} d \theta,
$$

where $\hat{\theta}$ is some small angle and we have selected the negative sign and eliminated it by interchanging the limits on the integral. It is well-known that the integral into (A.10) cannot be evaluated in terms of elementary functions. However, we could expand the integrand as a binomial series and integrate term by term [21, 22]. Alternatively, we can change the variables and convert the integral into the form of an elliptic integral [23]. Thus, according to [21] we have for (A.10)

$$
d \theta=\frac{2 k \cos z}{\sqrt{1-k^{2} \sin ^{2} z}} d z
$$

where the constant $k=\operatorname{def}\left(\theta_{0} / 2\right),|k|<1$, and $k \sin z=\operatorname{def} \sin (\theta / 2)$. Note that regarding the limits of the integral into (A.10), we have that for: i) $\theta=\theta_{0}$, then $z=\pi / 2$, and ii) $\theta=\hat{\theta}$, then $z=\hat{z}$.

It is well-known that three cases are distinguished by the regimes of total energy $E$ [24]: 1) Here $E<2 m_{1} g L$ is the energy required for the pendulum bob to reach the upper vertical position - finite motion; 2) $E=2 m_{1} q L$. Now, the energy level is critical and pendulum behavior is on special phase orbit $(\theta= \pm \pi)$ called separatrix (homoclinic one). It 
marks the boundary between oscillatory and hindered rotary motion of the pendulum; 3 ) Finally, $E>2 m_{1} g L$. The pendulum executes hindered rotary motion - infinite motion because the phase $\theta$ grows infinitely.

\section{A.2 Homoclinic solution of (16)}

Let us examine the equation (16) which has the form

$$
\ddot{\xi}_{1}-A(t) \xi_{1}-B\left(\theta_{0}\right)=0
$$

where $A(t)=\left(\frac{2 \omega}{c h(\omega t)}\right)^{2}+\frac{2 c l^{2}}{m_{1} L^{2}}, B\left(\theta_{0}\right)=\frac{2 g l^{2}}{L^{2}}\left(1-\cos \theta_{0}\right)+\frac{c}{m_{1}} \xi_{2}$ and $\xi_{2}=$ const. Without loss of generality we restrict our attention to the case when $\xi_{2}=0$. Moreover, according to Appendix 1 (and (13) also) $\theta_{0} \in[0, \pi]$ (see Fig. A1) and for $t \in[0.6,+\infty) A(t)=$ const.$>0$ (see Fig. A1). Thus, (A.12) is

$$
\ddot{\xi}_{1}-A \xi_{1}-B=0
$$

Hence, (A.13) is ODE from second order with constant coefficients.

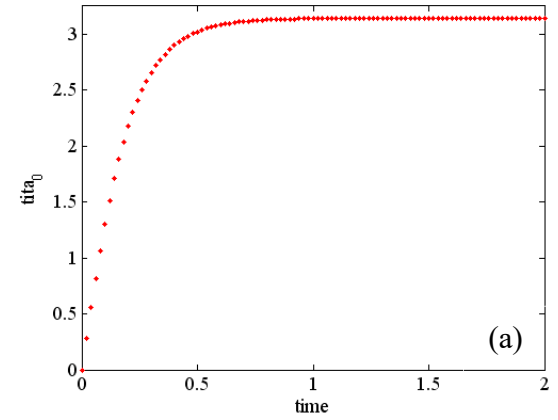

Fig. A1. Solutions for $\theta_{0}$ (a) and $A(t)$ $c=30[\mathrm{~N} / \mathrm{m}]$ and time $t$ is in seconds.

In the case when $l<<$, i.e. $\mu=(l / L)^{2} \approx 0$, we find that $B=0$ and (A.13) is a homogeneous linear ODE from second order

$$
\ddot{\xi}_{1}-\tilde{A} \xi_{1}=0
$$

with the general solution

$$
\xi_{1}(t)=C_{1} \operatorname{sh}(t \sqrt{\tilde{A}})+C_{2} \operatorname{ch}(t \sqrt{\tilde{A}}),
$$

where $C_{1}=\frac{1}{\sqrt{\widetilde{A}}} \dot{\xi}_{1}\left(t_{0}\right), C_{2}=\xi_{1}\left(t_{0}\right)$, and $\widetilde{A}=\left(\frac{2 \omega}{\operatorname{ch}(\omega t)}\right)$ are constants. 